\title{
Modelo mediacional de aprendizaje situado para investigación educativa
}

\author{
mirandaesquer72@hotmail.com
}

Se presentan procesos y resultados de una experiencia de innovación dentro de un programa de maestría. La aplicación de este modelo ha sido evaluada a partir de las percepciones de los participantes. La investigación se inscribe en la esfera epistemológica en un paradigma constructivista, mientras que a nivel metodológico asume un paradigma mixto. El método empleado fue investigación-acción con un diseño no experimental y uno de tipo fenomenológico, arrojando algunas conclusiones como que la construcción del objeto de estudio implica dos procesos simultáneos: vigilancia epistemológica y generación de obstáculos epistémicos.

Processes and results of a experience of innovation within a program of masters are presented. The application of this model has been evaluated from the point view of the perceptions of participants. This investigation is registered in the epistemologic sphere within a constructivist paradigm, whereas at a methodologic level it assumes a mixed paradigm. The method applied was investigation-action with a nonexperimental design and one of fenomenológic kind, drawing some conclusions as for instance that the Construction of the Object of Study implies two simultaneous processes: epistemologic monitoring and generation of epistemologic obstacles.

Este texto apresenta os procesos e resultados de uma experiência de inovação no interior de um programa de mestria. A aplicação deste modelo tem sido evaluada a partir das percepções dos participantes. A pesquisa, da qual se fala, inscrivese na esfera epistemológica dentro de um paradigma construtivista, enquanto a nivel metodológico assume um paradigma misturado. O método aqui empregado foi o chamado de pesquisa-ação usando um desenho não experimental e um outro do tipo fenomenológico o qual produciu algumas conclusões como por exemplo que a constução do objeto de estudo implica dois procesos ocorrendo simultáneamente: a vigilância epistemológica e a generação de obstáculos epistemológicos.
Mediación docente, aprendizaje in situ, investigación educativa.

Teaching mediation, in situ learning, educational research.
Mediação docente, aprendizagem in situ, pesquisa educativa.

* Maestro en Educación, Campo: Formación Docente por la Universidad Pedagógica Nacional, Unidad 26-B, Navojoa, Sonora, México. M.C. Universidad Pedagógica Nacional, Instituto Tecnológico de Sonora, Universidad del Valle de México. 
Necesitamos civilizar nuestras teorías, o sea, una nueva generación de teorías abiertas, racionales, críticas, reflexivas, autocríticas, aptas para autorreformarnos.

\section{Introducción}

\section{La Universidad Pedagógica Nacional, UPN,} como institución actualizadora y formadora de docentes, presenta dentro de su oferta educativa la Maestría en Educación, Campo: Formación Docente. Dicho programa educativo se desarrolla en cuatro semestres, de manera semi-escolarizada. Se integra por tres líneas temáticas: socio-histórica, psico-pedagógica y metodológica. Dentro de la línea metodológica se llevan a cabo cuatro seminarios, para formalizar la tesis de grado, siendo estos: Introducción a la Investigación Educativa, Seminario-Taller de Investigación I, Seminario de Tesis y Seminario de Investigación II.

Existe un bajo índice de titulación de esta Universidad, por lo que surge la idea inicial de esta investigación: posiblemente se deba a que no se han desarrollado de la mejor manera dichos seminarios de investigación, de tal forma que los alumnos no llegan a consolidar su documento recepcional. Como egresado del programa y conociendo de manera directa las estrategias de trabajo dentro de esta línea se identificaron las áreas de oportunidad que integrarían el Modelo Mediacional Aprendizaje Situado de Investigación Educativa, ASIE: antologías de trabajo, tutorías, construcción de tesis de grado, grupo de discusión virtual y mediación docente.

La presente investigación pretendió alcanzar el siguiente Objetivo General:

- Evaluar los distintos elementos del Modelo Mediacional ASIE a partir de las percepciones de los participantes.

Los Objetivos Particulares planteados fueron:

- Diseñar, desarrollar e implementar los recursos y estrategias del Modelo Mediacional ASIE

Desde la dimensión reflexiva del profesorado, Porlán concibe la mediación docente de manera activa ya que: "(...) desde la práctica reconstruye críticamente su propia teoría y participa, así, en el desarrollo significativo del conocimiento y la práctica profesional" (Porlán, 1997, p. 132).

Díaz Barriga y Hernández (2003) conceptualiza el Aprendizaje in situ, respecto al que afirma que se basa en el modelo contemporáneo de cognición situada que toma la forma de un aprendizaje cognitivo, el cual busca desarrollar habilidades y conocimientos propios de la profesión, así como la participación en la solución de problemas sociales o de la comunidad de pertenencia. Otra característica que agrega es que este tipo de aprendizaje, enfatiza la utilidad o funcionalidad de lo aprendido y el aprendizaje en escenarios reales.

Se adaptó la propuesta de Ballenilla (1995) sobre la evolución de los Modelos Didácticos, para interpretar la evolución de los modelos de Investigación Educativa, IE, de los participantes. Al respecto se presenta la siguiente figura:
Modelo IE
Modelo
Personal
ASIE
Modelo IE
Modificado

El Modelo IE Personal, de los participantes se caracterizaba por formas elementales de investigación, no sistemáticas ni reflexivas. Las experiencias de IE se circunscribían en todos los casos a sus trabajos de titulación de la Licenciatura, que iban desde Ensayos, hasta Tesis Documentales. El Modelo IE Personal estaba sobrecargado de estrategias y técnicas básicas como: revisión de listados, estadísticas descriptivas (sumatorias y medias, principalmente), consultas bibliográficas, entre otras.

El Modelo ASIE, es el Modelo de Referencia. Dentro de este Modelo se acentúa la sistematización y reflexión de los datos, la construcción y reconstrucción de los objetos de estudio, la colaboración entre los participantes dentro de los procesos constructivos, así como el empleo de metodologías cuantitativas o cualitativas.

Dentro de los diseños de investigación se retomaron no experimentales y cuasiexperimentales, a la vez que se abordaron técnicas de recolección como: el test y el 
cuestionario. Se les impartió un taller para calcular Ji cuadrada, t de Student, y Alfa de Cronbrach mediante el paquete SPSS v. 15, incorporando incluso los materiales en la antología de lecturas.

Revisando las metodologías cualitativas, se abordaron los siguientes métodos y diseños: el Método de Investigación-Acción con Diseños Etnográficos o Fenomenológicos, Fenomenología Hermeneútica con Diseño de Teoría Fundamentada. Se revisaron técnicas de recolección, análisis y de validación. Se les impartió un Taller para el manejo del programa Atlas Ti v. 5.0 para el análisis de textos, abordando de manera posterior, en las clases del Seminario, el análisis de fotografías mediante el mismo programa, y el software Transana, para la transcripción de audios y videos.

El Modelo IE Modificado ha incorporado los elementos del Modelo ASIE, que cada participante ha trabajado para el proceso de construcción de su objeto de estudio. Dentro de este Modelo Mediacional ASIE, enfatizamos dos procesos durante la construcción de su objeto de estudio:

a. De Vigilancia Epistemológica (Bourdieu, Chamboredon y Passeron, 2003) que nos permitió revisar en forma retrospectiva, los procesos de construcción de nuestro objeto de estudio: asumiendo un tanto la mirada del observador ${ }^{1}$.

b. De establecimiento de Obstáculos Epistémicos que nos permitió orientar de manera prospectiva el trabajo de construcción del objeto de estudio, retomando la postura del espíritu científico: más preguntas que respuestas (Bachelard, 1994), o desde la perspectiva del pensamiento complejo: más incertidumbres que certezas (Morin, 1999).

La aplicación de este Modelo ASIE, se esquematiza en la siguiente figura:

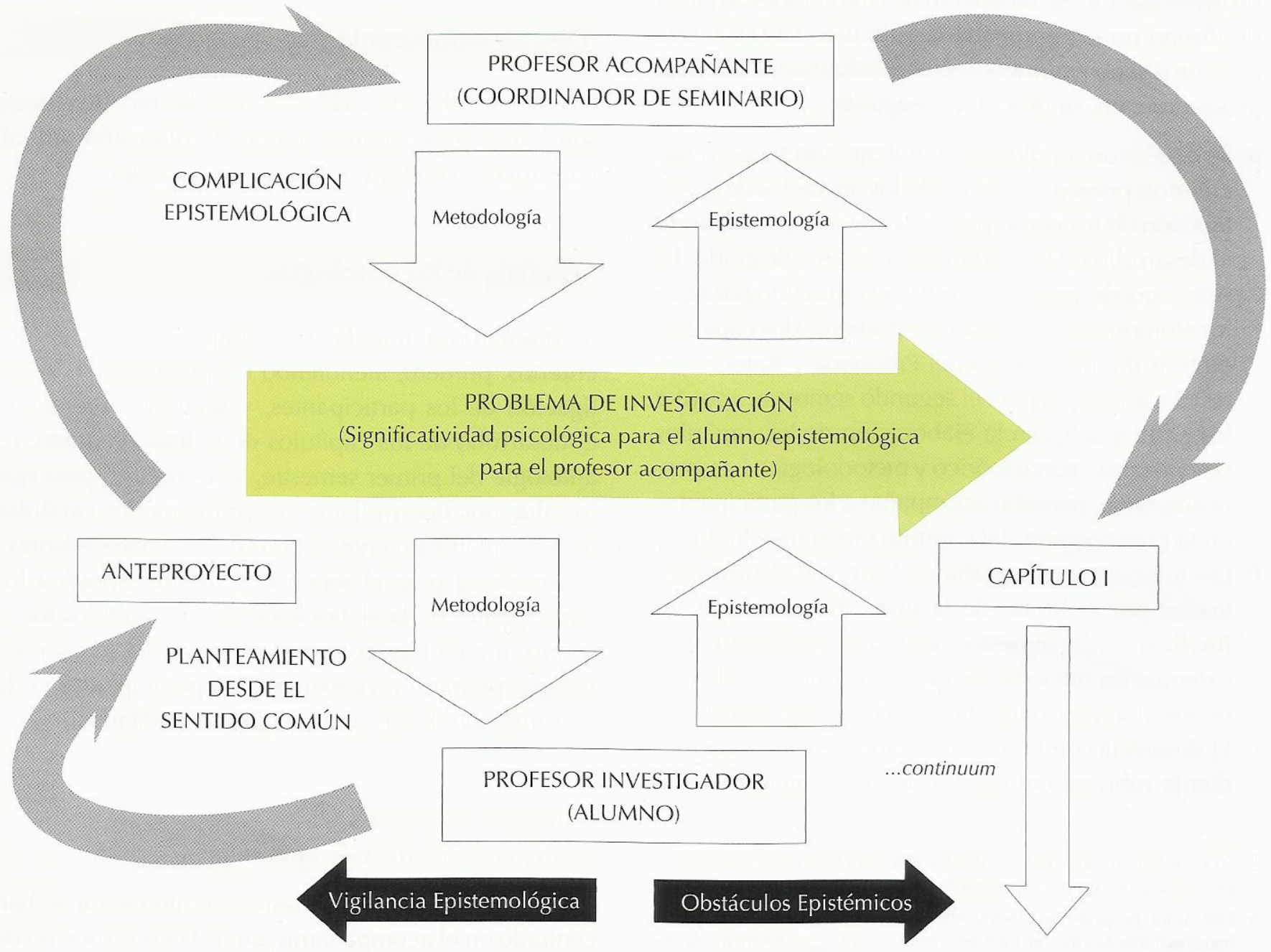

1 Desde la propuesta epistemológica de la Teoría del Campo Unificado. 
El problema de investigación de cada participante se trabajó en forma de espiral: se inició con un anteproyecto, planteado desde el sentido común; se intervino para complicar epistemológicamente el objeto de estudio, con lecturas apropiadas. Se construyó el primer capítulo de la tesis (planteamiento del problema) y así sucesivamente con el resto del capitulado. El profesor investigador revisaba retrospectivamente (vigilancia epistemológica) y prospectivamente (obstáculos epistémicos) la construcción de su objeto de estudio.

\section{Método}

A nivel epistemológico, se retomó un paradigma constructivista; mientras que a nivel metodológico, se concibió un paradigma mixto (Valles, 2003).

El método de investigación, es el conocido como Investigación-Acción (Elliott, 1996). Para los diseños de investigación, se recuperan dos tradiciones: aquella de diseño no experimental (Stenhouse, 1991)2 y otra más, de diseño fenomenológico (McKernan, 2002) $)^{3}$. El procedimiento, se describe enseguida:

a. Se diseñaron antologías de trabajo con base en dos criterios: primero, atendiendo los proyectos de investigación de los participantes, y segundo, retomando el desarrollo de los capítulos de la tesis de grado. La Antología del primer semestre, se estructuró para que los alumnos desarrollaran sus primeros dos capítulos de tesis (Planteamiento del Problema y Antecedentes). La antología para el segundo semestre, apoyó a los participantes en la elaboración de los capítulos tres y cuatro (marco teórico y metodología). La tercera antología, permitió acompañar a los participantes en la construcción del capítulo cinco (resultados).

b. Las tutorías, se vinculaban a las necesidades planteadas por el titular de la línea metodológica. Al finalizar cada semestre, el titular del Seminario, extendía un oficio de recomendaciones a cada uno de los alumnos, para que las retomaran en tutorías.

c. El desarrollo de la tesis de grado, se evaluaba mediante rúbricas entregadas desde el inicio de cada

2 En realidad Stenhouse, L., utilizaba diseños cuasi-experimentales y experimentales en sus investigaciones.

3 Este autor propone un diseño etnográfico, ya que sostiene que la Investigación-Acción, si bien es cierto, cuenta con técnicas de recolección de datos muy específicas, a la vez carece de las técnicas necesarias para el análisis de datos.
Seminario de Investigación, para que los participantes pudieran guiar su construcción.

d. Se implementaron cuatro técnicas de recolección de datos: cuestionario de encuesta, escritos reflexivos de los alumnos, diario de campo y grupo de discusión virtual, para monitorear el proceso de construcción de su tesis de grado, así como el papel de la mediación docente.

e. Se diversificaron las estrategias de trabajo durante el proceso constructivo: foros de presentación de avances, exposiciones, estudios de casos, visitas a biblioteca, visitas a ligas de internet, participación en grupo de discusión virtual, entre otras.

\section{Resultados}

Por cuestiones de espacio, se presentan únicamente los resultados del diseño fenomenológico.

\section{Diseño fenomenológico}

Las dimensiones de análisis abordadas en este reporte son: antologías, tutorías, grupo de discusión virtual, construcción de tesis y mediación docente.

\section{Análisis de las antologías}

Se diseñaron antologías de trabajo con base en dos criterios: primero, atendiendo los proyectos de investigación de los participantes, y segundo, retomando el desarrollo de los capítulos de la tesis de grado. La antología del primer semestre, se estructuró para que los alumnos desarrollaran sus primeros dos capítulos de tesis (planteamiento del problema y antecedentes). La antología para el segundo semestre, apoyó a los participantes en la elaboración de los capítulos tres y cuatro (marco teórico y metodología). La tercera antología, permitió acompañar a los participantes en la construcción de su capítulo quinto (resultados).

\section{Categoría de análisis}

percepción positiva de antología

Las antologías diseñadas hasta este momento, se han centrado en el acompañamiento de la construcción de las tesis de grado. En la siguiente cita se presenta el co- 
mentario de un estudiante sobre la antología elaborada en el segundo semestre:

La antología nos guía en cómo ir dándole forma a nuestra investigación y a cada uno de los capítulos, además que nos ayuda a enriquecernos con las tesis que se han realizado anteriormente sobre temas parecidos al nuestro.

Sobre la antología del tercer semestre, un participante del curso comentaba:

Me parecieron muy completas y se me hizo muy innovador la última que entregó en electrónico, así la puedo guardar en un disco y no hace tanto bulto como esas antologías de tres kilos que luego no hay donde guardarlas. De esta manera, si algún día ocupo información puedo recurrir al electrónico más rápido. También me gustó, porque aunque es "tutti frutti" pues nomás imprimo lo que ocupo y no ando cargando con toda la antología. También de paso tenía muy buena presentación esta última antología, se veía más formal y elaborada que aquellos bonches de copias de muchos libros, hasta puede hacer su patente para guía de maestría y que le pasen una feria. Esta muy bien, práctico, útil y formal.

En esta tercera antología, se optó por una versión electrónica de la misma, con hipervínculos a veinte libros electrónicos, en apoyo al desarrollo del capítulo de resultados. Retomando a Díaz-Barriga (2003), la funcionalidad de lo aprendido, está presente, pues coinciden los sujetos en que las antologías les apoyaron en la construcción de su capitulado.

Los elementos de transferibilidad y significatividad se desencadenaron mediante este apoyo, de la siguiente forma: al vincular la antología al capitulado en construcción se logró transferencia de lo que se aprendía a lo que se tenía que hacer, por otra parte; cuando las temáticas que integraban las antologías eran similares a las de sus proyectos podíamos establecer la significatividad epistemológica en este recurso.

\section{Categoría de análisis}

\section{percepción para el mejoramiento de la antología}

Los participantes marcaron un área en particular de mejora, para la antología del segundo semestre:
Lo que si le recomiendo es el tamaño de la letra, pues lastima mucho la vista, ojalá pudiera hacer algo al respecto

Este punto se corrigió para la tercera antología utilizada.

\section{Análisis de las tutorías}

Dentro del programa de postgrado se contempla el espacio de tutorías para acompañar de manera personal al participante en la construcción de su tesis. El número de tutorados por docente es en promedio cinco.

\section{Categoría de análisis \\ percepción positiva de tutorías}

En la siguiente cita, un estudiante comparte su percepción sobre las tutorías:

La frecuencia con la que he asistido es muy poca, pero las veces que se han llevado a cabo han sido provechosas y a la vez surgen dudas, en verdad se aclaran muchas de estas y no quiero que se cambie el tutor pues es la persona que me puede apoyar más con mi trabajo, pues sabe lo que se quiere lograr y conoce sobre el tema, y aunque si él no conociera con gran profundidad mi tema estoy seguro que haría lo posible por apoyarme, es un tutor que no sólo ayuda en la tutoría, sino que nos apoya en todo momento.

El alumno expone en su cita la empatía generada por su tutor, pues afirma que si no supiera sobre el tema, haría lo posible por apoyarlo. Al respecto De León (1996 citado en Durán, 2006) destaca la empatía como posibilidad para acceder a esa esfera particular y perceptual de los protagonistas y comunicar el entendimiento sensible del mundo de éstos, tal y como no lo vemos.

En otra cita, una alumna, comparte su percepción respecto a este espacio de construcción social:

(...) si uno como alumno no tiene esta claridad así te pongan de tutor al maestro más chicho (diría...) no se hará nada, o será como una hojita que se deja llevar por el viento pero al final no le encontrará sentido alguno a su trabajo, debo confesar que yo al inicio sabía qué quería hacer, mas no sabía cómo, se me presentaron algunas trabas para hacer lo que quería y perdí tiempo de cierta manera en encontrar ese sentido, iba 
a tutorías con C... y recuerdo, él me hablaba, hazlo de tal manera, lee tal cosa, y yo decía, de qué me va servir... (...) y este tercer semestre que encontré más claridad y sentido, veo que todo lo que me decía C... tenía algo que ver, porque finalmente lo retomé, y en tutorías cuando M... me decía, pues no te entiendo mucho pero haz esto y veremos que sale... me ayudó bastante para encontrar ese sentido que tanto necesitaba, y creo, espero, no pecar de soberbia, que tal vez con dudas, confusiones ahí va saliendo algo bueno e interesante.

Los tutores que la participante ha tenido, le apoyaron más en el aspecto metodológico -el cómo, en palabras de ella-, que en la parte teórica, debido a la naturaleza del tema -el duelo infantil-. Una característica importante en este caso, es la significatividad psicológica que representa el objeto de estudio para la alumna: sabía qué quería, pero no sabía cómo obtenerlo. Por otra parte para los tutores el objeto de estudio planteado por la maestrante, es significativo a nivel epistemológico: entender lo que la alumna quiere, entender la interpretación que la novel investigadora realiza sobre su objeto de estudio, es la principal preocupación de los tutores.

\section{Categoría de análisis área de mejora de tutorías}

En algunas ocasiones los participantes, compartieron posibles áreas de mejora de las tutorías. La siguiente cita, nos aclara un poco esta categoría:

¿Qué les parecieron las tutorías con sus respectivos tutores? ¿Cómo las podríamos mejorar? La verdad fui muy pocas veces, no hacía la gran cosa cuando iba, así que empecé a trabajar de mi cuenta esperando tener resultados para ir con algo que comentar. Eso sí, cuando tenía alguna duda iba con el maestro y me ayudaba. La verdad tenía otra idea de las tutorías, como que más apoyo por parte del tutor, se que el compromiso y el trabajo es mío pero lo sentía muy aparte.

Este caso ilustra, el desapego del tutor respecto al proyecto de investigación del alumno; y se coincide con los hallazgos de Durán (2006), cuando afirma que los alumnos de postgrado demandan mayor empatía de sus profesores.
De igual forma, la siguiente cita del corpus, expresa la poca implicación del tutor con el objeto de estudio de su tutorado.

Me gustaría que el asesor profundizara más en sus recomendaciones referentes a nuestras investigaciones, ya que siento que sus comentarios son muy por encima y no hay tanta ayuda de su parte.

El apoyo del tutor es superficial, lo que denota baja significatividad epistemológica, posiblemente porque el tutor conoce poco del tema que está acompañando. La paradoja de Durán (2006) sobre acompañamiento y desolación en este proceso, es interesante: podemos acompañarlos físicamente, pero experimentarán una desolación en la esfera emocional, cuando el tutorado no percibe algún interés por parte del tutor sobre el curso que tomará su proyecto de investigación.

\section{Construcción de tesis}

El proceso de investigación se les planteó desde el inicio, desde la perspectiva del espíritu científico de Bachelard (1994). A partir de este posicionamiento epistemológico la investigación no se agota hasta que se han contestado todas las preguntas planteadas, lo cual no es sinónimo de un proceso lineal, porque una interrogante resuelta trae consigo otra más.

\section{Categoría de análisis construcción del objeto de estudio, semestre III}

Este proceso de construcción de la tesis, lo detalla de manera significativa, una estudiante en este programa de postgrado.

La reconstrucción se me ha hecho más pesada, es como derribar y quitar escombros, por ejemplo, tuve que analizar tres veces las cincuenta y ocho fotos, porque la primera vez me salió un mundo de datos, algunos códigos repetitivos y demasiados específicos, la segunda fue para podar categorías y darle más forma a los datos, y la tercera para agrupar las categorías similares y encontrarles sentido con los fundamentos teóricos (...) He hecho modificaciones a los pri- 
meros capítulos, siguiendo las recomendaciones de mi tutor.

Estos días he tenido muchas dudas sobre las categorías, apliqué el análisis de abajo hacia arriba, quiero decir de los datos hacia la teoría, me han salido categorías no relevantes y no sé si eliminarlas o conservarlas porque no están en mi marco teórico.

El proceso de investigación es dialéctico: se avanza en plantear la pregunta, definir los objetivos, establecer los supuestos o las hipótesis, plantear la justificación del estudio, etc. Pero al revisar los antecedentes de su investigación, posiblemente se encuentra que alguien ya investigó sobre la temática; por lo que será necesario un nuevo planteamiento del problema. Desde esta lógica, se concibe cada capítulo de la tesis como vasos comunicantes: si se presentan cambios en un capítulo, implica cambiar los otros capítulos.

Pero en definitiva, el proceso de actualización en la UPN no es homogeneizante. Presentamos una cita, donde el alumno, no entendió lo que significaba estudiar un postgrado.

Se me hace una tontería, las tesis quedan, simplemente quedan, la tesis no es de una sola persona, el tutor da su aporte, la tesis es de dos, tutor o guía y el tutorado, si reprueba la tesis reprueba el tutor así de sencillo.

Desde la epistemología de Bachelard (1994) el espíritu conservativo del estudiante ha contestado sus preguntas -si alguna vez las hubo-, y desde esta posición epistemológica no es posible hacer ciencia. Por otra parte, la relación tutor-tutorado, la plantea en un nivel bastante simplista: ya Morin (2001) citado por Castro (2006) en Jacobo (2006) plantea la equivocación de esta idea relación lineal causa-efecto, de producto-productor.

Los postgrados no estamos exentos de este tipo de alumnos, que entran a ellos por un "papel" más, como lo afirma el mismo estudiante en la siguiente cita:

Ah y si averigua quien fue el responsable de este correo no me repruebe póngame por lo menos un siete, que sólo entré a la UPN por un papel sale $y$ vale.

Retomando a Ausubel (1987) esta actitud negativa del alumno no permitirá, promover algún tipo de aprendizaje significativo -recordemos que la actitud positiva del alumno, es un elemento central en la promoción de estos tipos de aprendizajes, por el docente-.

Antagónica a la postura conformista de la cita anterior, otra participante expone su posición al respecto:

Mi trabajo de tesis, soy psicóloga, ha sido un trabajo en ocasiones desgastante más que física mentalmente, por la magnitud de mi tema y sobre todo que yo soy parte de ese proceso de duelo, yo no lo llegué a pensar pero varias personas me lo dijeron que sería algo muy difícil el ser objetiva, el separarme del papel de tía para ser la investigadora, y como les dije a ellos y lo repito ahora, si es muy difícil, quitarte la camiseta y los sentimientos de tía, y ponerme el de investigadora que no siente, sobre todo cuando entrevistaba y tenía que hacer preguntas que yo sabía que podían tal vez hacer recordar algo doloroso a mi sujeto de estudio, pero si se puede, lo que me hacia sacar esa fuerza tal vez, y lo retomó para cuando tengo que hablar del tema y explicar mi tesis, es el acordarme lo difícil que ha sido este proceso, para mi sobrino y familia, (...)

La significatividad psicológica de su objeto de estudio, debido a que se revisa el proceso de duelo de un sobrino ante la pérdida de su madre -hermana de la investigadora-, ha implicado un triple proceso de vigilancia epistemológica, ya que la construcción de categorías se ha triangulado en investigadores (Latorre, 2005), para garantizar la objetivización de la subjetividad.

Al respecto, Habermas (1986 citado en Porlán, 1997, p. 126) apunta: "podemos considerar que sólo desde una epistemología que reconozca la dialéctica que se establece entre los intereses llamados subjetivos y el conocimiento pretendidamente objetivo, podremos comenzar a dar cuenta de un conocimiento que emancipe el pensamiento rutinario dominante, en la perspectiva de generar un pensamiento reflexivo y autónomo".

Otro alumno, narra este proceso de construcción de la tesis:

Ya al inicio del tercer semestre ante el inminente fin del ciclo escolar me vi apurado por hacer el plan de trabajo y aplicar, porque no tenía seguridad de tener un grupo similar al año siguiente. Este tercer semestre se me hizo muy apresurado la aplicación y elaboración de materiales, pues editaba los materiales, videos y otros para adap- 

tarlos a las clases. Me sentía presionado a llevar acabo y terminar la propuesta, así pronto se llegó al fin de ciclo y al día del foro donde presentamos resultados y conclusiones. Esto último lo sentí muy repentino, como sin pensarlo había llegado a un intento de cierre

Durante este tercer semestre de la Maestría en Educación, los alumnos presentaron un apartado de conclusiones preliminares; por lo que ese intento de cierre se trabajará de manera exhaustiva en el último semestre.

\section{Dimensión de análisis mediación docente}

Esta dimensión de análisis, centra la reflexión en torno a la actividad del coordinador de la línea metodológica. Esta mediación docente está determinada por el estilo docente, al respecto, Castro (2006, citado en Jacobo 2006, p. 83) sobre el mismo, afirma: "En principio se plantea que el estilo docente es una construcción que hace el profesor de su realidad de ser docente; la configuración del estilo tiene lugar en el sistema de interacción didáctica, en los flujos permanentes del interactuar, en las acciones recíprocas de ésta, que van modificando el comportamiento, tanto del profesor como del alumno y perfilan un estilo de ser docente, de frente a estilos diferentes de ser alumno".

Sobre este proceso de mediación docente, un alumno expresaba:

Me gustó el dinamismo en las clases, el no clavarnos en un sólo estilito de trabajo, a veces comentarios o reunirnos en equipo para buscar información, exponer ante el grupo los avances y comentarlo entre todos, creo que eso retroalimentó mucho.

El estilo del docente provoca encuentros a partir del estilo particular del estudiante, complementándose, a partir de la naturaleza de los mismos: profesores organizados y comprometidos, pueden establecer relaciones positivas con la misma clase de alumnos. No es así en la siguiente cita, donde otro estudiante protagoniza un lamentable desencuentro:

Ya sé, no le caigo bien (favor que me hace) y usted a mí tampoco, así con relación a los co- mentarios y sugerencias mejor le haré caso a R...y C... ah! tengo las sospechas que es rencoroso y vengativo por su egolatría así que lo estoy predisponiendo, ahí está mi trabajo, estoy en sus manos.

Castro (2006, citado en Jacobo 2006), sobre el estilo docente, afirma que este permanece en un estado constructivo de manera incesante alrededor de la interacción profesor-alumno y alumno-profesor. Es un proceso dialéctico de encuentros y desencuentros, de orden y desorden.

Un alumno más, nos brinda un punto de vista, sobre este tipo de desencuentros, que únicamente se pueden entender por la inmadurez de quienes lo protagonizan. Veamos:

En el grupo del que hago mención, por sus comportamientos tan juveniles en ocasiones, siento que no tenemos idea de la madurez que debemos tener en este nivel de estudio. Sí, nos interesa estudiar, pero me queda la sensación de que no nos ha caído el veinte del nivel que como maestrante debemos alcanzar o tener (...) Pero repito; no se muestra gran preocupación por aprender (no quiero decir que no hemos aprendido, porque en lo particular yo si he aprendido mucho), quizás la única materia que nos mueve el tapete es la del seminario de investigación.

Otro alumno, reconoce el trabajo de mediación docente que se ha realizado en la línea metodológica.

Creo y no me parece equivocarme que la metodología que está empleando con nosotros, así como el trabajo que estamos desarrollando está marcando una pauta en ambas sedes de la UPN, pues recuerdo que en el primer foro los de primer semestre no traían mucho, en cambio ahora tratan de alcanzar lo que nosotros mostramos el año pasado, me da la impresión de que Huatabampo no se quiere quedar atrás e intentan esforzarse aunque aún falta más.

De una o de otra manera, los profesores de esta línea de la UPN-Navojoa y UPN-Huatabampo, han retomado algunos elementos de este modelo mediacional (antologías, tutorías o construcción de la tesis), lo cual nos permitirá avanzar en los procesos de colegiabilidad de dichas universidades. 


\section{Dimensión de análisis grupo de discusión virtual}

Durante el segundo semestre se había contemplado el diario de campo, para que los alumnos desarrollaran las habilidades de observación y descripción mediante esta técnica. Una vez que el trabajo con esa técnica se decidió cerrar, se generó durante el tercer semestre un grupo de discusión virtual, disponible en la siguiente dirección electrónica: http://groups.google.com.mx/ group/profesores_reflexivos para que los alumnos reflexionaran en torno a dimensiones de análisis determinadas por el titular de la línea metodológica. El número de mensajes depositados al 4 de agosto de 2008 se presenta enseguida.

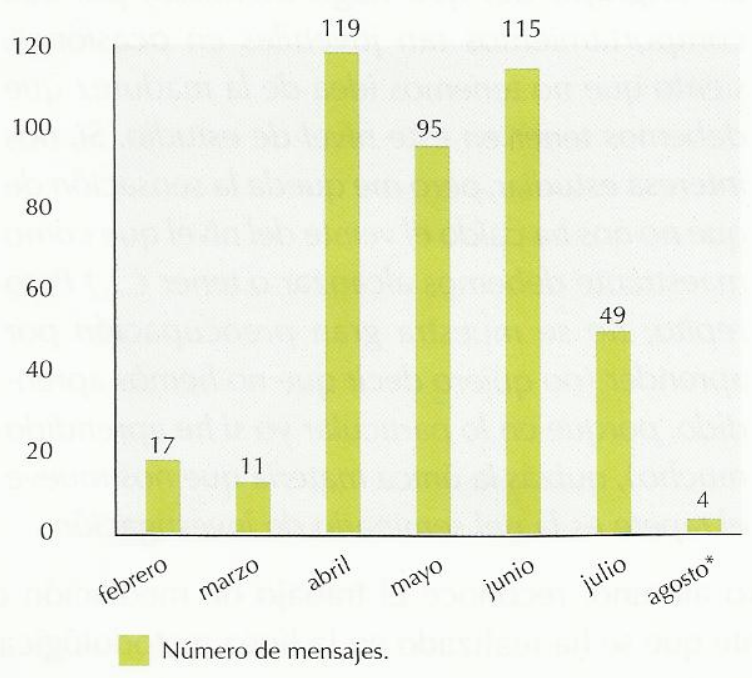

Se puede constatar que no obstante ser un período de receso vacacional, en julio los participantes dejaron cuarenta y nueve mensajes en la localidad electrónica del grupo, mientras que el total de mensajes fue de cuatrocientos catorce.

\section{Categoría de análisis percepción positiva sobre el grupo de discusión virtual}

Una participante, expresó su opinión respecto al grupo de discusión virtual y el avance cualitativo que representó, en comparación con el diario de campo que manejaban el segundo semestre.

El grupo de discusión me gustó más que el diario, no sé si porque me encanta navegar en internet jaja, pero siento que aquí me expreso de manera más amplia, si le digo que esto del grupo de discusión es como ir al psicólogo y lo mejor de todo sin costo, qué más se puede pedir. Me parece una muy buena técnica, la mayoría participó y creo que con todos pasó lo mismo de que aquí nos expresamos más libremente y más extenso. El grupo me parece algo padre, más novedoso, y sirvió de cierta manera para conocer un poquito más de nosotros mismos y los compañeros, ya que al diario sólo tenía acceso usted, y aquí todos nos leemos, todos sabemos las dudas de todos, sobre todo muy enriquecedor, y también comentar que esto hizo que estuviéramos más en contacto con usted, cuando no se podía de manera personal recurríamos a internet, por lo menos yo considero que la comunicación entre usted y nosotros ha mejorado bastante y sobre todo que tenemos una realimentación de su parte y a veces hasta de los compañeros.

La alumna expresa el avance en la comunicación entre docente-alumnos, a pesar de que los encuentros eran tres por semana. Dentro de este grupo se subieron vínculos a sitios de investigación local, nacional e internacional, así como libros electrónicos que los alumnos solicitaban para el desarrollo de sus proyectos de investigación.

\section{Categoría de análisis percepción áreas de mejoramiento del grupo de discusión virtual}

Sin embargo, desde el punto de vista del alumno, esta técnica es factible de mejorarse.

Me parece buena estrategia, pero en lo personal no me funcionó tanto, porque a veces no tenía acceso a internet donde estaba y para cuando revisaba el correo ya tenía muchos sin contestar, hubo una época en que llegaban todos muy seguidos (...) Algo bueno de este grupo es que permite mantener el diálogo posterior al cierre del seminario. Como canal de comunicación me pareció muy bueno.

El asunto de la falta de conectividad, en algunos estudiantes, es una limitante para este tipo de estrategias virtuales; sin embargo, es recomendable un seguimiento virtual junto con el acompañamiento presencial en los estudiantes de postgrado. 


\section{Conclusiones}

A partir de los datos recabados podemos establecer las siguientes conclusiones iniciales:

- Las Antologías para la línea de investigación del programa de Maestría deben actualizarse de manera continua, retomando aquellas lecturas que permitan construir los distintos capitulados de las tesis.

- Las tutorías deben realizarse con un plan de trabajo preestablecido entre el tutorado y el tutor, tomando en cuenta las indicaciones de los coordinadores de la línea metodológica.

- Se debe avanzar en la especialización de los tutores en ciertas líneas temáticas para poder acompañar de manera más informada a los tutorados a su cargo.

- La construcción del objeto de estudio no es un proceso lineal, es un proceso dialéctico, con avances y retrocesos dentro del proceso de construcción del capitulado de la tesis de grado.
- Es necesario implicar dos procesos simultáneos en este proceso de construcción: vigilancia epistemológica -de manera retrospectiva- y obstáculos epistémicos -en forma prospectiva-.

- La mediación docente, debe ser estratégica, lo cual implica desarrollar significatividad epistemológica hacia los proyectos de investigación de los estudiantes, diversificando las estrategias de trabajo.

- Esta mediación docente, está condicionada por el estilo docente: siendo a su vez condicionado por los distintos estilos de los estudiantes.

- El grupo de discusión virtual fue una estrategia relevante para promover la empatía entre el docente y los alumnos.

- El Modelo Mediacional ASIE promueve aprendizajes situados en los estudiantes, ya que los aprendizajes son significativos, transferibles y contextualizados.

Elliott, J. (1996). El cambio educativo desde la Investigación Acción. Madrid: Morata

Jacobo, H. (2006). Educación y formación de profesores. Complejidad cognitiva y entorno global. Barcelona: Ed. Pomares.

López, R. (2002). Educación y Cultura Global. Valores y Nuevos Enfoques Educativos en una Sociedad Compleja. México: SEPYC-UAS.

Latorre, A. (2005). La investigación-acción. Barcelona: Ed. GRAO.

Mckernan, J. (2002). Investigación-acción y currículum. Madrid: Ed. Morata.

Porlán, R. (1997). Constructivismo y escuela. Sevilla: Díada. Stenhouse, L. (1991). Investigación y desarrollo del currículum. Madrid: Ed. Morata.

Valles, M. (2003). Técnicas cualitativas de investigación social. Madrid: Ed. Síntesis. 


\section{Diálogo del conocimiento}

En hora buena comenzamos a preocuparnos por un tema que se ha convertido en verdadera "caja negra" cuando se habla de la formación de posgrado: la pertinencia de los cursos de metodología de la investigación. El dilema que se plantea es el de apropiarse el estudiante de los métodos de los profesores especialistas que investigan en las líneas que ofrece la Maestría, aprendiendo en el contacto directo con ellos, en sus Seminarios temáticos, en sus búsquedas y en su manera de ver el mundo o acudir a una profundización en los paradigmas, metodologías, técnicas de recolección de información y su relación con el Proyecto de tesis que desea desarrollar.

El profesor Jesús Bernardo Miranda presenta el resultado de una investigación en la Universidad Pedagógica Nacional de México, que partió de este tipo de preocupaciones. Aunque se refiere a la necesidad de mantener una "vigilancia epistemológica" del proceso de construcción de conocimiento científico y habla de investigar in situ los "obstáculos epistemológicos" que enfrenta el estudiante real en su proceso de elaboración de la tesis, considero que su propuesta es la puesta a prueba de un modelo de intervención didáctico para recomponer la relación tutor-posgraduando.

Es el Modelo Mediacional de Aprendizaje Situado para investigación educativa, ASIE, que se compone de: antologías de trabajo en cada una de las etapas de la investigación -sesiones de tutoría con el especialistaconstrucción de la tesis de grado -grupo de discusión virtual- y mediación docente. Cada una de estas etapas son presentadas en una apretada síntesis que nos deja con ganas de saber más al respecto y entender mejor algunos términos, por ejemplo: ¿Es lo mismo "antología" que estado del arte o revisión de literatura? ¿O solamente se hace antología de las tesis ya elaboradas sobre el mismo tema? ¿Existe alguna guía o manual para la tutoría o se da en la relación espontánea entre los agentes? ¿Por qué si el Modelo apunta a superar las dificultades para la elaboración de la tesis, hay una unidad de análisis llamada "Construcción de tesis de grado"? ¿Cuál es la diferencia entre la mediación docente y la tutoría? ¿Qué se entiende por "rúbricas"?

Pero más allá de estas preocupaciones semánticas, es de resaltar la preocupación principal del tema y el tratamiento que se le da al enfoque metodológico de la investigación misma. Es muy importante partir de la manera como cada estudiante asume la metodología para, desde allí presentarle el modelo de intervención didáctica innovador, acompañarlo en su aplicación y después dar cuenta de las transformaciones ocurridas en el proceso gracias a lo que llama "aprendizaje situado", vale decir reconstruyendo la relación entre la significatividad de la lógica de la investigación y la psicologización de las experiencias individuales en el estudiante.

La investigación abre entonces el tema de la pertinencia de la formación en metodología y muestra que con una planificación intencionada, fundamentada en la teoría del aprendizaje situado, es posible mejorar el proceso de construcción de la tesis y elevar los niveles de posgraduación.

De todas maneras, el trabajo del profesor Miranda nos estimula para iniciar investigaciones sobre los procesos de investigación desde el punto de vista de los sujetos y los obstáculos psicológicos y epistemológicos que hay que caracterizar y remover si queremos conformar comunidades científicas.

Alfonso Tamayo Valencia 